\title{
Lossless medical image compression through lightweight binary arithmetic coding
}

Joan Bartrina-Rapesta, Victor Sanchez, Joan SerraSagristà, Michael W. Marcellin, Francesc Aulí-Llinàs, et al.

Joan Bartrina-Rapesta, Victor Sanchez, Joan Serra-Sagristà, Michael W. Marcellin, Francesc Aulí-Llinàs, lan Blanes, "Lossless medical image compression through lightweight binary arithmetic coding," Proc. SPIE 10396, Applications of Digital Image Processing XL, 103960S (19 September 2017); doi: $10.1117 / 12.2273725$

Event: SPIE Optical Engineering + Applications, 2017, San Diego, California, United States 


\title{
Lossless Medical Image Compression through lightweight Binary Arithmetic Coding
}

\author{
Joan Bartrina-Rapesta ${ }^{\mathrm{a}}$, Victor Sanchez ${ }^{\mathrm{b}}$, Joan Serra-Sagristà ${ }^{\mathrm{a}}$, Michael W. Marcellin ${ }^{\mathrm{c}}$, \\ Francesc Aulí-Llinàs ${ }^{\mathrm{a}}$, and Ian Blanes ${ }^{\mathrm{a}}$ \\ ${ }^{a}$ Department of Information and Communications Engineering, Universitat Autònoma de \\ Barcelona, ETSE, E-08193 Cerdanyola del Vallès, SPAIN \\ ${ }^{\mathrm{b}}$ Department of Computer Science, University of Warwick, CV4 7AL, Coventry, UK \\ bElectrical and Computer Engineering,University of Arizona, Tucson, AZ 85721-0104, USA.
}

\begin{abstract}
A contextual lightweight arithmetic coder is proposed for lossless compression of medical imagery. Context definition uses causal data from previous symbols coded, an inexpensive yet efficient approach. To further reduce the computational cost, a binary arithmetic coder with fixed-length codewords is adopted, thus avoiding the normalization procedure common in most implementations, and the probability of each context is estimated through bitwise operations. Experimental results are provided for several medical images and compared against state-of-the-art coding techniques, yielding on average improvements between nearly 0.1 and 0.2 bps.
\end{abstract}

Keywords: Medical Image Compression, CCSDS-123, Lossless Coding, Arithmetic Coding.

\section{INTRODUCTION}

Thanks to medical imaging, physicians observe organs, bones, soft tissue and blood vessels with clarity. The observation inside of the human body allows radiologists to more easily diagnose pathologies such as cancer, infectious diseases, appendicitis, cardiovascular diseases, trauma or musculoskeletal disorders, among others. ${ }^{1}$

Two of the main techniques employed during the medical acquisition data process are X-ray and magnetic resonance. One of the most common sources of X-ray medical images is Computed Tomography (CT). CT images are acquired with ionized X-rays which yield a collection of 2D images (slices) that combined with sophisticated algorithms to produce three-dimensional (3D) images. Other X-ray image types are the General X-ray Angiography (GXA), which present blood vessels in several body parts like arms, legs, foots, etc.; and Coronary Angiogram Video Sequences (CAVSs), which focus on coronary vessel trees for diagnosing cardiovascular diseases. On the other hand, Magnetic Resonance Imaging (MRI) does not employ ionizing radiation during the acquisition process, making it useful for imaging the brain.

To efficiently store and transmit medical images, mostly lossless image compression has been adopted by the physician community as an appropriate technique since it allows perfect reconstruction of the data employed during the medical diagnoses. ${ }^{2}$ The Digital Imaging and Communications in Medicine (DICOM) standard ${ }^{3}$ is typically adopted to store and distribute medical images in Picture Archiving and Communications Systems (PACS). ${ }^{4}$ The DICOM standard supports various compression image techniques such as JPEG, ${ }^{5}$ JPEG-LS, ${ }^{6}$ JPEG2000, ${ }^{7}$ and H.264. ${ }^{8}$ To this end, several research contributions have been based on these coding techniques. ${ }^{9-14}$ For example, in ${ }^{12}$ the authors define an inter-slice coding in JPEG-LS. In, ${ }^{9-11}$ H.264 is tailored for lossless compression of 3D and 4D medical images based on the observation that slices of these high-dimensional data may be treated as frames of video sequences. The work in ${ }^{13}$ presents a method for region of interest coding for compression of digital mammography based on the scalability properties of JPEG2000. In, ${ }^{14}$ an estimator is defined to evaluate the effectiveness of employing inter-slice transforms in CT images compressed with JPEG2000. This work proposes to employ a lightweight arithmetic encoder for compressing medical images with higher efficiency than current coding techniques included in DICOM.

Further author information: (Send correspondence to Joan Bartrina-Rapesta)

E-mail: joan.bartrina@uab.cat, Telephone: +34 935811777

Applications of Digital Image Processing XL, edited by Andrew G. Tescher, Proc. of SPIE

Vol. 10396, 103960S · C 2017 SPIE · CCC code: $0277-786$ X/17/\$18 $\cdot$ doi: 10.1117/12.2273725

Proc. of SPIE Vol. 10396 103960S-1 


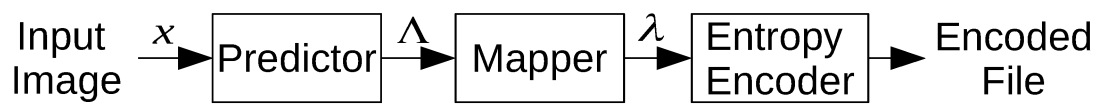

Figure 1. CCSDS-123 encoding scheme.

Lossless image compression is a very transverse research topic, as demonstrated by its use not only for medical images, but also for remote sensing. However in the remote sensing scenario, compression techniques are mainly developed to operate on-board either satellites or airplanes, where computational resources and memory are limited. In this context, The Multispectral \& Hyperspectral Data Compression Working Group with the Consultative Committee for Space Data Systems (CCSDS) ${ }^{15}$ is in charge of proposing techniques for remote sensing data compression. Such techniques are mainly developed to be implemented on board, where limited resources are available and low complexity encoders are needed. In 2012, CCSDS published its latest standard, CCSDS-123.0-B-1, ${ }^{16}$ focused on lossless compression for multispectral and hyperspectral images based on prediction. In what follows, we will refer to CCSDS-123.0-B-1 as CCSDS-123.

In this paper, we make use of the CCSDS-123 predictor. To this end, we substitute the current entropy encoder of the CCSDS-123 by a lightweight contextual arithmetic coder. The proposed encoder utilizes inexpensive operations to estimate probabilities, does not incorporate renormalization, and employs a simple context model. This work extends our previous contribution ${ }^{17}$ that employs a scanning order based on line-by-line and bitplaneby-bitplane, estimates the probability of the current symbol using only bitwise operations, and employs at most three causal neighbors from the current slice for modeling the context. In this work the probability is estimated as proposed in, ${ }^{17}$ however the samples are scanned in slice-by-slice and bitplane-by-bitplane. Additionally, the context modeling employees the closest four causal neighbors at most. We specifically concentrate on lossless compression of grayscale anatomical medical images, such as CTs, MRIs, GXAs, and CAVS.

The rest of this paper is organized as follows: Section 2 briefly reviews the CCSDS-123 coding system, Section 3 describes the proposed context-based arithmetic coder with bitwise probability estimation, Section 4 explains how the proposed arithmetic coder is incorporated in a coding scheme that uses the predictor of CCSDS123, Section 5 shows experimental results, and Section 6 concludes this work.

\section{CCSDS-123 COMPRESSION}

The CCSDS-123 standard, which is limited to encoding samples of $N=16$ bits per pixel per slice, can be structured in three stages: Predictor, Mapper and Entropy Encoder. Fig. 1 illustrates the encoding pipeline of CCSDS-123.

In summary, the predictor estimates the value of the current sample $x_{i, j, k}$ using previously scanned samples. This predicted sample is denoted by $\widetilde{x}_{i, j, k}$. The prediction error $\Lambda$ is computed as

$$
\Lambda_{i, j, k}=x_{i, j, k}-\widetilde{x}_{i, j, k}
$$

and then mapped to a non-negative integer $\lambda_{i, j, k}$ called the mapped prediction residual. Here, $i$ and $j$ represent spatial coordinate indices, while $k$ is the slice index. The entropy encoder is in charge of encoding $\lambda_{i, j, k}$ without loss. For the entropy encoder in CCSDS-123, one can choose between a sample- or a block-adaptive encoder. The sample-adaptive encoder achieves better performance than the block-adaptive encoder when the signal is encoded at more than 1 bit per sample (bps). However, because the minimum codeword length of the sampleadaptive encoder is one bit, block-adaptive encoding yields superior performance for signals that can be encoded at less than 1 bps.

Further details of the CCSDS-123 stages can be found in ${ }^{18}$ and. ${ }^{19}$ 


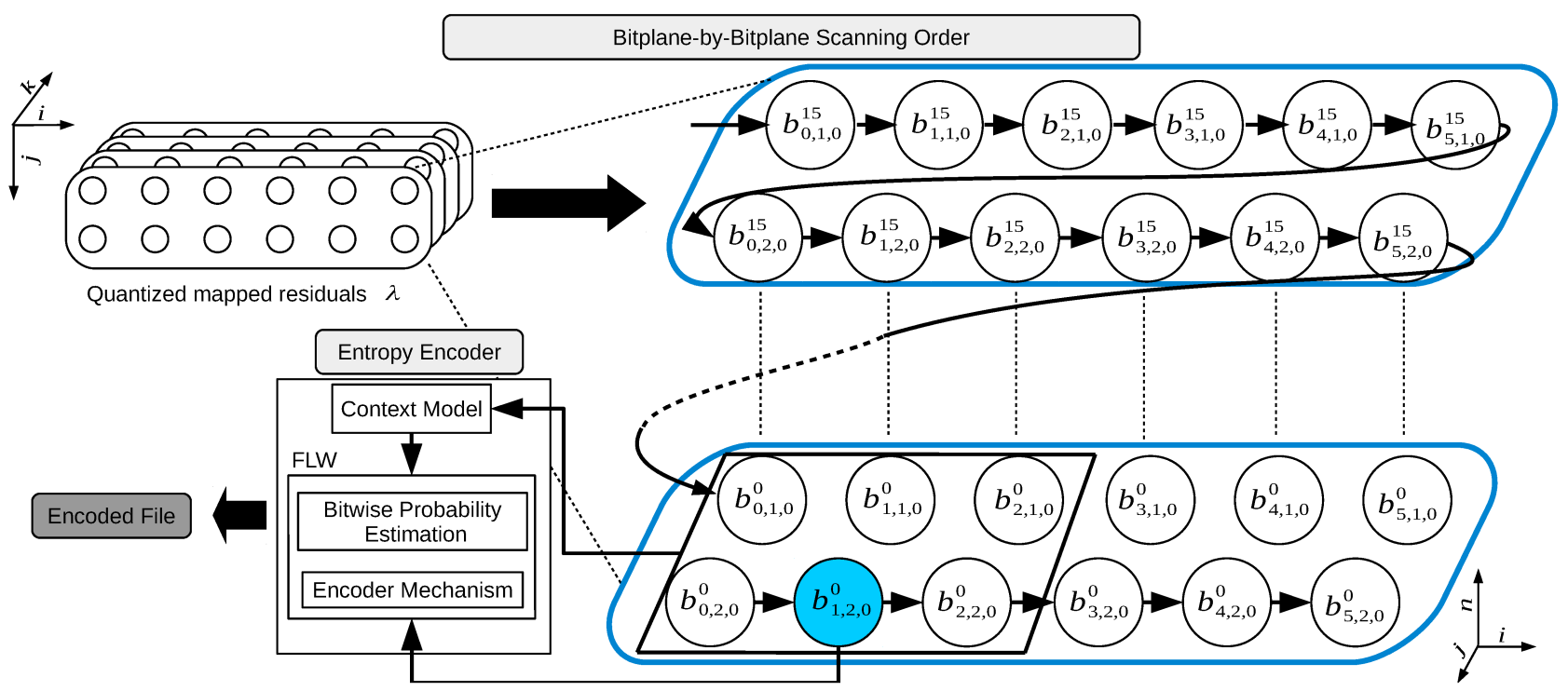

Figure 2. Illustration of the scanning order and the entropy encoder.

\section{A LIGHTWEIGHT BINARY ARITHMETIC CODER WITH CONTEXT MODEL}

Context-based arithmetic encoders typically obtain excellent coding performance at all rates, however they are not included in CCSDS-123 because they can have a high computational demand owing to: a) context formation, b) probability estimation, and c) the renormalization procedure, which are expensive operations and are executed intensively.

The entropy encoder presented in this paper works with binary symbols. To this end, we denote the $n$-th bit of the binary representation of $\lambda_{i, j, k}$ by $b_{i, j, k}^{n}$, with $N-1 \geq n \geq 0$ and $b_{i, j, k}^{N-1}$ representing the most significant bit. Here, $N$ is chosen to provide a sufficient number of bits to represent all the $\lambda_{i, j, k}$.

Our proposal processes data in a slice-by-slice fashion. Once a slice is predicted, and the residuals are mapped to positive values, it is entropy encoded on a bitplane-by-bitplane basis. The entropy encoder makes use of context model patterns obtained using a context window that contains symbols coded previously to the current symbol. The top left portion of Fig. 2 displays the remapped prediction residuals $\lambda$, organized by slice. The binary representation for the first slice $(k=0)$ is shown on the right, while the bottom left portrays the entropy encoder, which is fed by the current bit to be encoded and its context. The bit to be encoded is shaded in blue, while the context window is framed with a rectangle.

\subsection{Proposed Context Model}

Let $\mathbf{M}$ be the set of all possible patterns that can occur within the context window, with context $m \in \mathbf{M}$ being a particular realization, resulting in a context index $c \in \mathbf{C}=\{0, \ldots, C-1\}$. These context indices (loosely referred to as contexts in what follows) are determined by a modeling function $F: \mathbf{M} \rightarrow \mathbf{C}$. For each bit $b$ to be coded, a probability model is used, corresponding to its context $c$. In particular, the probability model estimates the conditional probability $p(b \mid c)=p(b \mid F(m))$. After encoding, the probability model is updated with the latest coded bit $b$. That is, $p(b \mid c)$ is estimated on the fly. Specifically, our probability model estimates the probability $p(b=0 \mid c)$.

A careful design of the context model is required to obtain high coding efficiency. This task is complicated by the goal of not increasing the computational load excessively.

Our simple strategy for context modeling employs a context window that contains at most four causal neighbors as depicted in Fig. 3. We consider several choices for the context modeling function $F$. The first ignores all samples within the context window except the one directly above the sample of interest. This is 


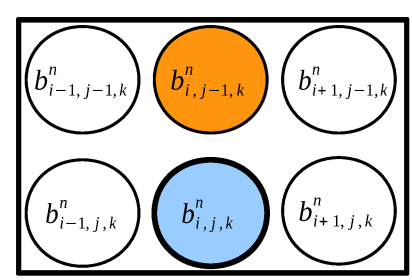

(a) $\mathrm{V}$

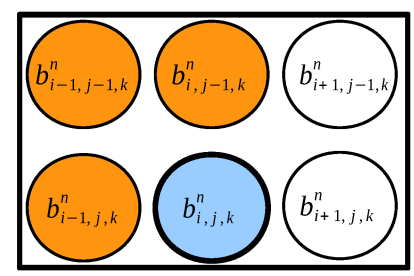

(d) HVDL

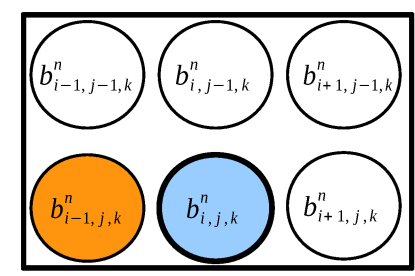

(b) $\mathrm{H}$

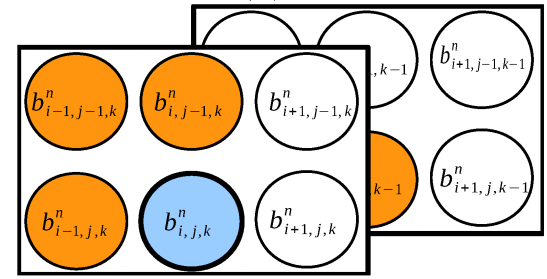

(e) HVDLS

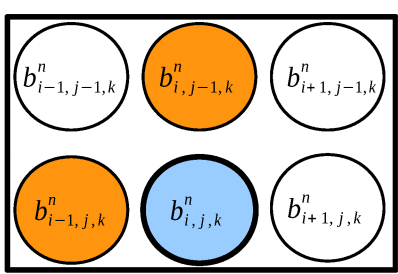

(c) $\mathrm{HV}$

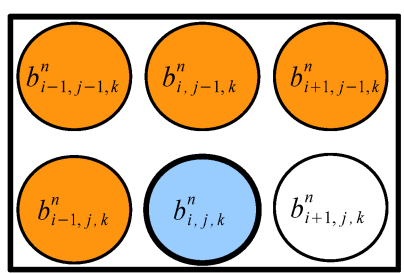

(f) HVDLDR

Figure 3. Illustration of the context models employed to encode the $b_{i, j, k}^{n}$ symbol.

Table 1. Context assignments employing 4 causal adjacent neighbors with HVDLDR context model.

\begin{tabular}{ccccccccccccccccc}
\hline$s_{i-1, j, k}^{n}$ & 0 & 1 & 0 & 1 & 0 & 1 & 0 & 1 & 0 & 1 & 0 & 1 & 0 & 1 & 0 & 1 \\
$s_{i-1, j-1, k}^{n}$ & 0 & 0 & 1 & 1 & 0 & 0 & 1 & 1 & 0 & 0 & 1 & 1 & 0 & 0 & 1 & 1 \\
$s_{i, j-1, k}^{n}$ & 0 & 0 & 0 & 0 & 1 & 1 & 1 & 1 & 0 & 0 & 0 & 0 & 1 & 1 & 1 & 1 \\
$s_{i+1, j-1, k}^{n}$ & 0 & 0 & 0 & 0 & 0 & 0 & 0 & 0 & 1 & 1 & 1 & 1 & 1 & 1 & 1 & 1 \\
\hline$c$ & 0 & 1 & 2 & 3 & 4 & 5 & 6 & 7 & 8 & 9 & 10 & 11 & 12 & 13 & 14 & 15 \\
\hline
\end{tabular}

indicated in Fig. 3 (a). The next three choices are shown in Fig. 3 (b), (c) and (d). The pneumonics V, H, and DL are used to denote neighbors in the figure, where $\mathrm{V}$ (Vertical) denotes the sample above the bit to be encoded, H (Horizontal) denotes the sample to the left, and DL (Diagonal Left) denotes the sample to the left and above. To take advantage of dependencies between slices, the preceding slice $k-1$ can be included in the context window. In this case, the pneumonic S (Slice) denotes the co-registered sample in the previous slice. This sample is employed with HVDL as is shown in Fig. 3 (e). The last modeling function evaluated employs the four closest causal samples within a slice as depicted in Fig. 3 (f), resulting in the HVDLDR context model, where DR (Diagonal Right) denotes the sample to the right and above.

Rather than the actual bit (from bitplane $n$ ) of each neighboring sample, the so-called "significance state" is employed to compute the context $c$. To this end, let $s_{i, j, k}^{n}$ denote the significance state of the sample at location $i, j, k$ at bitplane $n$. A value of 1 indicates that the sample contains a 1 at bitplane $n$ or higher. Table 1 shows how $c$ is derived from the significance states of the neighbors for the HVDLDR modeling function. Experimental results for all context modeling functions are presented in a subsequent section.

The entropy coder and its associated probability models are initialized only once at the beginning. In particular, the initial probability model for each context is set to a value of $p(b=0 \mid c)=0.66$. The probability is biased towards 0 since, as found empirically, bits of higher bitplanes have higher probability of being 0 , thus allowing FLW to adapt faster.

\subsection{Bitwise Probability Estimation}

Contributions aimed to reduce the computational load by estimating probabilities using multiplication-free implementations can be found in the literature. ${ }^{20,21}$ One of the latest contributions to low complexity arithmetic coding presents a context-adaptive binary arithmetic coder with fixed-length codewords (FLW) ${ }^{22}$ which reduces complexity by avoiding the renormalization procedure. FLW outperforms the $\mathrm{MQ}^{23}$ and $\mathrm{M}$ coders ${ }^{21}$ in terms of coding performance, but still estimates probabilities via division. 


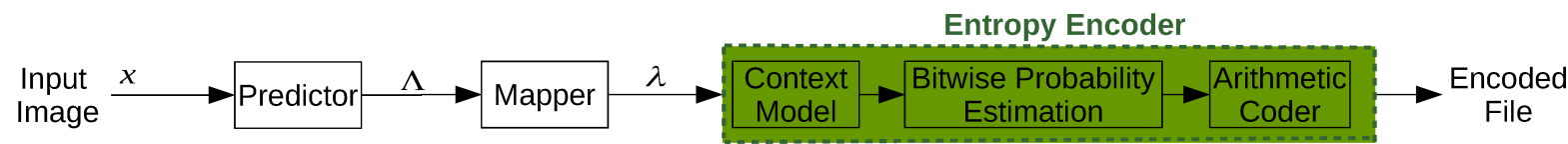

Figure 4. Adopted coding approach.

The arithmetic encoder used in this paper is devised to reduce the computation related with probability estimation and the renormalization simultaneously. To this end, our contribution utilizes inexpensive operations to estimate probabilities, does not incorporate the renormalization procedure, and employs a simple context model. It yields strong coding performance for different medical imagery. Our probability estimation procedure builds on that of FLW.

Originally, FLW uses a sliding window to estimate the probability of the symbols coded using a division operation. In ${ }^{17}$ the sliding window size of FLW was adapted to deal only with power of two sizes, which allows the use of low complexity bitwise operations and avoids the need for division. Very roughly, for each context $c$, FLW uses a sliding window of symbols coded with that context. The length of this window varies between $\mathcal{T}$ and $2 \mathcal{T}-1$ symbols, and the probability estimate is updated once every $\mathcal{V}$ symbols coded. The substitution of the division by bitwise operations requires that $\mathcal{V}=\mathcal{T}$ and that both are a power of two.

Further details of how the bitwise operations are defined for FLW can be found in. ${ }^{17}$

\section{LIGHTWEIGHT MEDICAL IMAGE CODING PROPOSAL}

The proposed arithmetic coder is incorporated in a lossless coding scheme, providing improved compression performance over current lossless compression techniques included in the DICOM standard. Although the novel entropy encoder presented here may be incorporated in any coding system, we employ it in the CCSDS-123 coding pipeline. Fig. 4 illustrates the adopted coding approach, which employs the predictor and mapper of CCSDS-123, but substitutes the usual CCSDS-123 encoder by our entropy encoder (highlighted in green). The experimental results indicate that our proposed approach improves lossless compression ratios, on average, over those obtained by CCSDS-123, JPEG-LS, JPEG2000, and HEVC. ${ }^{24}$ Regarding specific video coding techniques, only results for HEVC are reported, since it is considered the successor of H.264.

\section{EXPERIMENTAL RESULTS}

This section presents a set of experiments aimed at the analysis and evaluation of the adopted coding scheme. Two different sets of experimental results are reported. In the first one, the proposed context modeling functions are evaluated in terms of coding performance in bits per sample (bps). And, in the second one, the resulting proposed overall approach is compared in terms of lossless compression performance with CCSDS-123, JPEG-LS, JPEG2000 and HEVC. For our proposal, the bitwise probability estimation employs $\mathcal{T}=\mathcal{V}=2^{12}$.

In, ${ }^{25}$ the impact of different CCSDS-123 parameters that control the operation of the prediction and the entropy encoder were evaluated, suggesting that correct parameter selection had more impact on the predictor stage than in the entropy encoder stage. Among the predictor parameters, local sum type, prediction mode, number of prediction bands, and predictor adaption rate were the most critical. In this work, the number of prediction bands $P$ is set to 3 , the predictor adaptation rate $\nu_{\max }$ is also set to 3 , and the predictor mode and local sum used are Neighbour Oriented and Full mode, respectively for all images.

For the experiments conducted in this work, we have selected a set of images collected with different medical devices. Image types and their main features are listed in Table 2. The reported values are the image type and name, the size of each image, its bitdepth and the first order entropy; which represents the entropy of individual samples, without accounting for any dependencies among pixels within or between components. The last column of Table 2 depicts a thumbnail of a single slice of each image type. 
Table 2. Summary of the data used in the experimental results. Image type, image name, image dimension $(I \times J \times K)$, bitdepth of the samples, first-order entropy (in bits per sample), and a thumbnail of a single slice of each image type.

\begin{tabular}{|c|c|c|c|c|c|}
\hline Image type & Image name & $(I \times J \times K)$ & Bitdepth & Entropy & Thumbnail \\
\hline Coronary Angiogram & cavs_1 & $512 \times 512 \times 64$ & 12 & 10.52 & \\
\hline Video Squences & cavs_2 & $512 \times 512 \times 82$ & 12 & 10.45 & \\
\hline (CAVS) & cavs_3 & $512 \times 512 \times 74$ & 12 & 10.13 & \\
\hline Computed & ct_1 & $512 \times 512 \times 48$ & 12 & 8.97 & \\
\hline Tomography & ct_2 & $512 \times 512 \times 56$ & 11 & 9.35 & \\
\hline$(\mathrm{CT})$ & ct_3 & $512 \times 512 \times 61$ & 12 & 9.23 & \\
\hline Magnetic Resonance & mri_1 & $256 \times 256 \times 100$ & 8 & 5.43 & \\
\hline Imaging & mri_2 & $512 \times 416 \times 20$ & 12 & 7.26 & \\
\hline (MRI) & mri_3 & $512 \times 512 \times 11$ & 8 & 6.19 & \\
\hline General X-ray & gxa_1 & $1024 \times 1024 \times 14$ & 12 & 9.67 & \\
\hline Angiography & gxa_2 & $1024 \times 1024 \times 5$ & 12 & 10.11 & \\
\hline (gxa) & gxa_3 & $1024 \times 1024 \times 4$ & 12 & 9.40 & \\
\hline
\end{tabular}

\subsection{Context Modeling Function}

The context model is used to select the probability model that is employed to encode the current symbol. Table 3 provides the lossless compression performance (in bps) resulting from each of the different context formations defined in Section 3.1, i.e., V, H, HV, HVDL, HVDLS, and HVDLDR. All results are produced using the bitwise probability estimator having $\mathcal{V}=\mathcal{T}=2^{12}$. Results from the table suggest that: 1) all of the modeling functions provide significant improvements over the pixel entropy reported in Table $2 ; 2$ ) differences in performance between the modeling functions are generally small; 3) the worst performance on average is achieved when a single sample is employed for the context function, i.e., context models $\mathrm{V}$ and $\mathrm{H} ; 4$ ) the best performance is obtained when four samples are used in the context function, i.e., context models HVDLS and HVDLDR; which yield an improvement of 0.05 and $0.04 \mathrm{bps}$ compared to $\mathrm{V}$ and $\mathrm{H}$ context formation, respectively. 5) when four context samples are employed, DR samples provide slightly more benefits than S samples. 
Table 3. Lossless coding performance (in bps) for the context modeling functions denoted by V, H, HV, HVDL, HVDLS, HVDLDR.

\begin{tabular}{|c|c|c|c|c|c|c|}
\cline { 2 - 7 } \multicolumn{1}{c|}{} & \multicolumn{5}{|c|}{ Context Formation } \\
\hline Image Name & $\mathrm{V}$ & $\mathrm{H}$ & HV & HVDL & HVDLS & HVDLDR \\
\hline \hline cavs_1 & 6.633 & 6.633 & 6.629 & 6.624 & $\mathbf{6 . 6 1 6}$ & 6.619 \\
cavs_2 & 6.355 & 6.355 & 6.351 & 6.348 & $\mathbf{6 . 3 4 2}$ & 6.345 \\
cavs_3 & 6.586 & 6.586 & 6.583 & 6.580 & $\mathbf{6 . 5 7 4}$ & 6.577 \\
\hline \hline ct_1 & 7.023 & 7.019 & 7.004 & 6.994 & 6.997 & $\mathbf{6 . 9 8 6}$ \\
ct_2 & 7.813 & 7.809 & 7.800 & 7.796 & 7.796 & $\mathbf{7 . 7 9 0}$ \\
ct_3 & 5.105 & 5.062 & 4.967 & 4.923 & 4.902 & $\mathbf{4 . 8 9 2}$ \\
\hline \hline mri_1 & 3.439 & 3.432 & 3.423 & 3.414 & $\mathbf{3 . 3 9 0}$ & 3.395 \\
mri_2 & 4.236 & 4.215 & 4.193 & 4.181 & 4.181 & $\mathbf{4 . 1 7 2}$ \\
mri_3 & 3.035 & 3.013 & 2.944 & 2.893 & 2.872 & $\mathbf{2 . 8 5 8}$ \\
\hline \hline gxa_1 & 5.887 & 5.887 & 5.879 & 5.873 & 5.869 & $\mathbf{5 . 8 6 8}$ \\
gxa_2 & 5.856 & 5.856 & 5.846 & 5.840 & 5.837 & $\mathbf{5 . 8 3 5}$ \\
gxa_3 & 6.030 & 6.030 & 6.013 & 6.002 & 5.993 & $\mathbf{5 . 9 9 2}$ \\
\hline \hline Average & 5.667 & 5.658 & 5.636 & 5.622 & 5.614 & $\mathbf{5 . 6 1 1}$ \\
\hline
\end{tabular}

\subsection{Lossless compression techniques comparison}

The results reported here compare the lossless performance of the proposed approach with JPEG2000, JPEG-LS, HEVC and CCSDS-123.

For JPEG2000, results are reported when the inter-slice redundancy is not exploited (2D) and when it is. To exploit the inter-slice redundancy the $5 / 3$ Integer Wavelet Transform (IWT) with 5 levels is employed among the slices. In both cases the codeblock size is $64 \times 64$. Regarding HEVC, intra and inter prediction with RExt extensions are enabled. For JPEG-LS, the slices are treated independently. JPEG2000 and JPEG-LS are DICOM compliant techniques, and although HEVC is not included in the DICOM standard it considered here since it provides appreciable improvements in coding efficiency compared to H.264. ${ }^{26}$ For the CCSDS-123 and our proposed coding technique the prediction is conducted employing neighbor oriented mode and using 3 previous bands.

The lightweight binary arithmetic coder with context model that is used in the proposed scheme employs the context model HVDLDR and the bitwise probability estimator having $\mathcal{V}=\mathcal{T}=2^{12}$.

The results reported in Table 4 indicate that for most cases, our proposed method using HVDLDR context formation outperforms JPEG2000, JPEG-LS, HEVC and CCSDS-123. Paying attention to the average results, our proposal attains a coding improvement of 0.19 bps over JPEG2000, 0.12 bps over IWT+JPEG2000, 0.18 bps over JPEG-LS, 0.26 bps over HEVC, and 0.12 and 0.20 bps over the sample- and block-adaptive modes of CCSDS-123, respectively.

\section{CONCLUSIONS AND FUTURE WORK}

Medical imaging facilitates diagnosis by radiologists thanks to the high resolution of the images acquired. The high resolution directly translates to more data to be stored and/or transmitted. For this reason the need to compress this data efficiently is always a topic of interest. 
Table 4. Lossless compression results for the proposed approach. For comparison, results for JPEG200, JPEG-LS, and HEVC are included. Results are reported in bits per sample (lower is better).

\begin{tabular}{|c|c|c|c|c|c|c|c|}
\hline \multirow{2}{*}{ Image Name } & \multicolumn{2}{|c|}{ JPEG2000 } & \multirow{2}{*}{ JPEG-LS } & \multirow{2}{*}{ HEVC } & \multicolumn{2}{|c|}{ CCSDS-123 } & \multirow{2}{*}{ Proposed } \\
\cline { 6 - 7 } & 2D & IWT & & & Sample & Block & \\
\hline \hline cavs_1 & 6.925 & 6.805 & 6.823 & 7.001 & 6.680 & 6.851 & $\mathbf{6 . 6 1 9}$ \\
cavs_2 & 6.674 & 6.514 & 6.611 & 6.735 & 6.423 & 6.591 & $\mathbf{6 . 3 4 5}$ \\
cavs_3 & 6.911 & 6.768 & 6.798 & 6.960 & 6.647 & 6.821 & $\mathbf{6 . 5 7 7}$ \\
\hline \hline ct_1 & 7.115 & 7.222 & 7.089 & 7.154 & 7.071 & 7.219 & $\mathbf{6 . 9 8 6}$ \\
ct_2 & 8.015 & 8.067 & 7.908 & 7.909 & 7.843 & 8.025 & $\mathbf{7 . 7 9 0}$ \\
ct_3 & $\mathbf{4 . 7 4 4}$ & 4.812 & 5.009 & 5.247 & 5.287 & 5.140 & 4.892 \\
\hline \hline mri_1 & 3.449 & 3.319 & 3.260 & $\mathbf{3 . 2 2 8}$ & 3.474 & 3.397 & 3.395 \\
mri_2 & 4.118 & 4.314 & 4.406 & 4.852 & 4.283 & 4.434 & $\mathbf{4 . 1 7 2}$ \\
mri_3 & 2.784 & 2.973 & $\mathbf{2 . 8 4 0}$ & 3.213 & 3.069 & 3.008 & 2.858 \\
\hline \hline hemo_1 & 6.339 & 5.961 & 6.272 & 6.066 & 5.870 & 6.049 & $\mathbf{5 . 8 6 8}$ \\
hemo_2 & 6.319 & 5.978 & 6.249 & 6.038 & 5.851 & 6.037 & $\mathbf{5 . 8 3 5}$ \\
hemo_3 & 6.311 & 6.087 & 6.273 & 6.099 & $\mathbf{5 . 9 8 4}$ & 6.157 & 5.992 \\
\hline \hline Average & 5.809 & 5.735 & 5.795 & 5.875 & 5.736 & 5.811 & $\mathbf{5 . 6 1 1}$ \\
\hline
\end{tabular}

In this contribution, we focused on the CCSDS-123 predictor followed by a lightweight contextual arithmetic encoder. Specifically, we proposed a set of context functions employing causal samples for coding medical images and estimating the probabilities needed by the arithmetic coder employing only bitwise operations instead of the division. These choices make our contribution efficient in terms of coding performance but cheaper in terms of computational load. We evaluate our proposal with different types of medical images such as Coronary Angiogram Video Sequences, Computed Tomography Imagery, Magnetic Resonance Imaging, and General X-ray Angiography; and compare in terms of lossless coding performance our proposal with different coding techniques such as JPEG-LS, JPEG2000, HEVC and CCSDS-123.

Experimental results indicate that, on average, our proposal improves over the current standard version of CCSDS-123 for lossless coding by nearly 0.1 bps. Compared with the coding techniques evaluated in this work our proposal provides improvements from 0.05 to 0.5 bps for JPEG2000, from 0.1 to 0.41 bps for JPEG-LS, from 0.1 to 0.68 bps for HEVC, and from 0.003 to 0.26 bps for CCSDS-123. These results suggest that the proposed compression scheme has great potential to be included in DICOM.

In addition, the results indicate that the use of the four closest neighbors for the context formation is enough to properly exploit the contextual information of medical images in an arithmetic encoder when the data to be encoded are obtained from the predictor of CCSDS-123. But as more samples are employed for the context formation the improvements increase.

\section{ACKNOWLEDGMENTS}

This work was supported in part by the Spanish Ministry of Economy and Competitiveness (MINECO) and by the European Regional Development Fund (FEDER) under Grant TIN2015-71126-R, by the Catalan Government under Grant 2014SGR-691, and by Centre National d'Études Spatiales (CNES). 


\section{REFERENCES}

[1] American Cancer Society (ACS) (June 2017).

[2] Schelkens, P., Munteanu, A., Barbarien, J., Galca, M., Giro-Nieto, X., and Cornelis, J., "Wavelet coding of volumetric medical datasets," IEEE Transactions on Medical Imaging 22, 441-458 (March 2003).

[3] Digital Image and Communication in Medicine, "DICOM," (May 2016).

[4] Cao, X. and Huang, H., "Current status and future advances of digital radiography and PACS," IEEE Eng. in Medicine and Biology Magazine 19, 80-88 (Sept. 2000).

[5] "Information technology - digital compression and coding of continuous-tone still images: Requirements and guidelines," (Feb. 1994).

[6] ISO/IEC, "JPEG-LS lossless and near-lossless compression for continuous-tone still images," (1999).

[7] "Information technology - JPEG 2000 image coding system - Part 1: Core coding system," (Dec. 2000).

[8] "H.264 - advanced video coding for generic audiovisual services," (May 2003).

[9] Sanchez, V., Nasiopoulos, P., and Abugharbieh, R., "Lossless compression of 4d medical images using h.264/avc," in [2006 IEEE International Conference on Acoustics Speech and Signal Processing Proceedings], 2, II-II (May 2006).

[10] Sanchez, V., Abugharbieh, R., and Nasiopoulos, P., "Symmetry-based scalable lossless compression of 3D medical image data," IEEE Trans. Medical Imaging 28(7), 1062-1072 (2009).

[11] Sanchez, V., Nasiopoulos, P., and Abugharbieh, R., "Novel lossless fmri image compression based on motion compensation and customized entropy coding," IEEE Transactions on Information Technology in Biomedicine 13, 645-655 (July 2009).

[12] Miaou, S., Ke, F., and Chen, S., "A lossless compression method for medical image sequences using JPEG-LS and interframe coding," IEEE Trans. Information Technology in Biomedicine 13, 818-821 (Sept. 2009).

[13] Bartrina-Rapesta, J., Serra-Sagrista, J., and Auli-Llinas, F., "Jpeg2000 roi coding through component priority for digital mammography," Computer Vision and Image Understanding 115(1), 59 - 68 (2011).

[14] Munoz-Gómez, J., Bartrina-Rapesta, J., Marcellin, M. W., and Serra-Sagristà, J., "Correlation modeling for compression of computed tomography images," IEEE Journal of Biomedical and Health Informatics 17, 928-935 (Sept 2013).

[15] Consultative Committee for Space Data Systems (CCSDS).

[16] "Lossless Multispectral \& Hyperspectral Image Compression." CCSDS-123.0-B-1 Blue Book, May 2012.

[17] Bartrina-Rapesta, J., Blanes, I., Auli-Llinas, F., Serra-Sagrista, J., Sanchez, V., and Marcellin, M., "A lighweight contextual arithmetic coder for on-board remote sensing data compression," IEEE Transactions on Geoscience and Remote Sensing, In Press. (2017).

[18] Blanes, I., Magli, E., and Serra-Sagrista, J., "A tutorial on image compression for optical space imaging systems," IEEE Geoscience and Remote Sensing Magazine 2, 8-26 (Sept 2014).

[19] Lossless Multispectral \& Hyperspectral Image Compression. CCSDS-120.2-G-1 Green Book, Dec 2015.

[20] Slattery, M. and Mitchell, J., "The Qx-coder," IBM Journal of Research and Development 42, 767-784 (Nov. 1998).

[21] Marpe, D., Schwarz, H., and Wiegand, T., "Context-based adaptive binary arithmetic coding in the H.264/AVC video compression standard," IEEE Transactions on Circuits and Systems for Video Technology 13, 620-636 (July 2003).

[22] Auli-Llinas, F., "Context-adaptive binary arithmetic coding with fixed-length codewords," IEEE Transactions on Multimedia 17, 1385-1390 (Aug. 2015).

[23] Taubman, D. S. and Marcellin, M. W., [JPEG2000 Image compression fundamentals, standards and practice], Kluwer Academic Publishers, Norwell, Massachusetts 02061 USA (2002).

[24] "H.265 - high efficiency video coding," (Apr. 2015).

[25] Auge, E., Sanchez, J. E., Kiely, A., Blanes, I., and Serra-Sagrista, J., "Performance impact of parameter tuning on the ccsds-123 lossless multi- and hyperspectral image compression standard," Journal of Applied Remote Sensing 7(1), 074594-074594 (2013).

[26] Sullivan, G. J., Ohm, J. R., Han, W. J., and Wiegand, T., "Overview of the high efficiency video coding (hevc) standard," IEEE Transactions on Circuits and Systems for Video Technology 22, 1649-1668 (Dec 2012). 